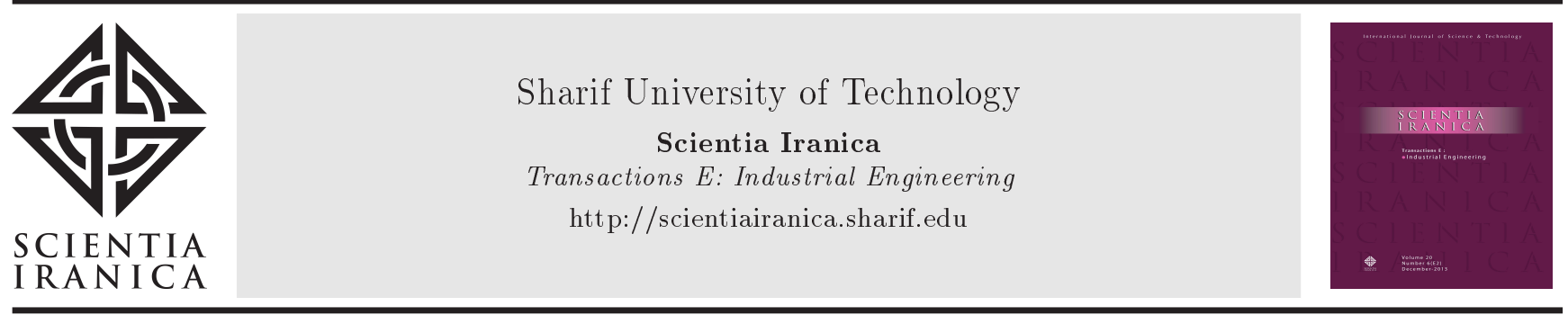

\title{
The surgical case scheduling problem with fuzzy duration time: An ant system algorithm
}

\author{
R. Behmanesh ${ }^{\mathrm{a}}$, M. Zandieh ${ }^{\mathrm{b}, *}$, and S.M. Hadji Molana ${ }^{\mathrm{a}}$ \\ a. Department of Industrial Engineering, Science and Research Branch, Islamic Azad University, Tehran, Iran. \\ b. Department of Industrial Management, Faculty of Management and Accounting, Shahid Beheshti University, G.C., Tehran, Iran.
}

Received 3 March 2017; received in revised form 6 October 2017; accepted 23 June 2018

KEYWORDS
Surgical case
scheduling;
Ant system;
Operating theater;
Fuzzy duration time;
Makespan;
Mixed integer
programming.

\section{Introduction}

Healthcare industries have been growing in the last decades and with the passing of time, costs of this large industry will increase. Based on statistics, healthcare expenditure of US in 2007 was estimated at $16.2 \%$ the gross domestic product [1] and it will reach $19.5 \%$ US GDP by 2017 [2]. On the other hand, Operating Rooms (ORs) are considered as the engine and the most critical causes of carrying the highest

\footnotetext{
*. Corresponding author.

E-mail addresses: rezaehs@yahoo.com (R. Behmanesh); m_zandieh@sbu.ac.ir (M. Zandieh); molana@srbiau.ac.cir (S.M. Hadji Molana)
}

doi: $10.24200 /$ sci.2018.20602 costs for a hospital; in many hospitals, more than $40 \%$ of costs come from several resources of surgery and ORs [3]. Therefore, it seems essential to improve OR management and patient flow by optimized sequencing and assigning available resources of ORs to patients. As a result, planning and scheduling play a crucial role in OR management and thereby, in recent decades, many researchers and practitioners have been attracted to the study of operating room scheduling problem.

In healthcare, a "surgical schedule" is arranged by determining the sequence of surgical cases and assigning them to operating rooms, surgeons, nurses, etc. in order to optimize objectives such as utilization, idle time, overtime, etc. [4]. Operating room scheduling generally deals with strategic, tactical, and operational problems [5-8].

The problem referred to in this paper is called 
surgical case scheduling and classified in operational level. In the literature on the operating room scheduling problems [9], a "Surgical Process Scheduling (SPS) problem" is divided into two sub-problems called "advance scheduling" and "allocation scheduling". The first sub-problem solves the planning step by determining some future dates for surgical cases in medium time horizon. The next level, namely "Surgical Case Scheduling (SCS) problem," as the second part of SPS solves scheduling step, which determines the start time and resource allocation of cases over a short time horizon (typically a day). It must be noted that allocation scheduling is within the scope of SCS problem, whereas advance scheduling is not. In some researches [1015] both sub-problems were addressed, while in other studies [2,16-22], the researchers reviewed the SCS problem.

Surgical process is divided into three subprocesses including pre-operative/surgery, perioperative/surgery, and post-operative/surgery for which various parameters are considered as input to each stage $[16,18,21]$. In the literature, upstream units comprise Pre-operative Holding Units (PHUs) and wards, while Post Anesthesia Care Units (PACUs) and Intensive Care Units (ICUs) are put in downstream units. Multiple operating rooms connect upstream to downstream units $[5,21,23-25]$. Also, the patients are divided to elective (inpatient and outpatient) and non-elective (urgent and emergency) cases. An elective case is the patient that is scheduled in advance by determining multi-resources as well as the start time of the case, while an emergency case that may arrive randomly on the day of surgery requires to be performed online in the same day. To measure operating room planning and scheduling, 8 main performance indicators have been used in the literature, namely waiting time, utilization, leveling, throughput, patient deferrals, makespan, preferences, and financial measures [26]. Also, there are three well-known scheduling strategies/booking systems that dedicate time of ORs to surgeon groups, namely open scheduling strategy, block scheduling strategy, and modified block scheduling strategy. In block scheduling strategy, a set of time slots is allocated to every surgery in a special group, typically in cyclic timetable (some weeks) manner. Surgical cases are scheduled in these time slots and they cannot be released. In contrast to block scheduling, in open scheduling strategy, surgical cases are scheduled on First-Come-First-Service (FCFS) and assigned to available ORs at the surgeon's convenience. In the other policy, namely modified block scheduling, block scheduling is modified by combining strategies of both block and open scheduling in order to enhance flexibility of strategy $[6,18,19]$.

In the literature on operating room scheduling, deterministic conditions are considered for scheduling and therefore, some deterministic models are constructed for this problem [21], while some conditions such as unpredicted incidents related to loss of resources, incidents in the surgical case, etc. increase duration of surgeries in operating room in real practice. This has motivated practitioners and researchers $[27,28]$ to focus on this problem under uncertain conditions and propose new algorithms to tackle uncertain operating room scheduling problem.

The following sections are organized as follows. In Section 2, we present various studies on operating room planning and scheduling regarding the scope of our problem. In Section 3, the problem is stated and then, the mathematical model for fuzzy surgical case scheduling is built. In Section 4, we propose fuzzy ant system algorithm in order to achieve fuzzy ant solution. Section 5 provides illustrative examples and computational experiments; finally, we conclude and present our suggestions for future research in Section 6 .

\section{Literature review}

Some researches $[5,7,26,29]$ provide the review of the most recent literature on operations research within the scope of surgery planning and scheduling problems. The major discussion in some studies relates to uncertain conditions in operating room scheduling and planning at three levels. Therefore, first, some papers which model this problem using deterministic times are reviewed and then, new researches that tackle the problem under uncertainty are addressed.

Xiang et al. [21] formulated a surgical case scheduling problem as multi-resource FJSP by using mixed integer linear programming model in order to minimize makespan during a day as they observed similarities between operating theater scheduling and FJSP. The authors considered sequence of operations (pre-operative, peri-operative, and post-operative) for each elective surgery in FJSP under open scheduling policy, and the deterministic durations were provided in all stages. They elaborated on Ant Colony Optimization (ACO) approach with a two-level hierarchical graph to integrate sequencing jobs and allocating resources at the same time to solve their model. It should be noted that they used variation coefficient of working time for all resources in their analysis so as to evaluate their ACO algorithm. Meskens et al. [19] addressed a multi-objective surgical case scheduling problem. The researchers applied genetic algorithm to tackle the problem by minimizing makespan, minimizing overtime hours, and maximizing desiderata of the surgical team. Moreover, block scheduling system and deterministic duration of surgery were assumed for operating rooms.

Lamiri et al. [30] addressed surgery planning problem assumed as SPS in planning level. Assigning elec- 
tive cases to different periods over a planning horizon was done in order to minimize two objectives consisting of the sum of costs related to the elective patients and overtime costs of operating rooms. The authors modeled the problem under uncertain condition. First, a novel stochastic mathematical programming was proposed and then, the Monte-Carlo simulation and MIP model were integrated to tackle the problem. In another advanced study, Lamiri et al. [31] addressed the same previous problem, and proposed and compared several optimization approaches in order to minimize expected overtime costs and patient-related costs, simultaneously, under stochastic condition. The authors applied Monte-Carlo simulation, MILP model, and meta-heuristics to solve the problem. Saremi et al. [32] proposed simulation-based optimizations in order to tackle outpatient case scheduling or SCS problem by providing stochastic service time data from a major Canadian hospital. The researchers modeled the problem to minimize multiple objectives including waiting time of the patient, completion time of the patient, and the number of surgery cancellations. Their first approach was Simulation-based Tabu Search (STS), which integrated discrete-event simulation and tabu search to schedule surgical cases. The second and third methods were Integer Programming Enhanced Tabu Search (IPETS) and Binary Programming Enhanced Tabu Search (BPETS). IPETS and BPETS were improved on STS by combination with integer programming and binary programming models, respectively. Lee and Yih [27] introduced a fuzzy scheduling strategy to find the start time of surgical cases in operating theaters as an SCS problem with uncertainty. The authors formulated this problem as flexible job shop with fuzzy sets. Resources of downstream, such as PACU and service time, were applied as constraints to their model. The researchers considered multiple objectives consisting of waiting time of the patient in the process flow, clinical resource idling, and total completion times in order to evaluate their model. The genetic algorithm was applied to solve the problem in two phases. In the first step, the relative order of surgical cases was determined and then, in the second stage, definite start time of surgical cases was found. A Monte-Carlo simulation was conducted to assess the schedules obtained and then, the results were compared with those of the previous studies with simulation based scheduling. It was concluded that the new approach outperformed the traditional approach. Marques and Captivo [28] focused on the surgical case assignment in a Portuguese hospital in order to optimize the use of the available surgical resources, and improved equity and access to operated and waiting elective patients. The researchers dealt with the problem in two stages; first, some patients from a large waiting list were selected to be scheduled for surgery; then, a day, an operating room, and a time block were allocated to the patients. To tackle the problem, three deterministic MILP models were constructed concerning the administration intention, surgeons, and a halfway between administration and surgeons; finally, they proposed a robust approach concerning the OR and surgeon occupation time for each surgery under uncertainty. The authors developed deterministic models in uncertain conditions using the approaches in the literature. In another work conducted by Mateus et al. [33], elective surgeries scheduling problem was studied in a Portuguese public hospital. The authors developed local search heuristics to tackle different versions of the problem. In this study, the problem was divided into two sub-problems; first, patients were selected from a waiting list for surgery and then, a day, an operating room, and a time block were assigned to patients. The researchers evaluated the proposed algorithms for a real case compared with MILP models.

As it was described, the nature of the proposed surgical operations problem, especially durations of service times, in all stages is considered certain. However, as given in the literature $[27,30,32]$, duration times in operating theaters are not precise and, due to uncertain conditions of service time for operations in the SCS problem, it would be better to insert probabilistic data into this problem. On the other hand, if uncertainty is taken into account for duration time parameters, the complexity of the problem will be increased. Dubois et al. [34] stated that we were permitted to use either probabilistic distributions or fuzzy numbers in order to represent uncertainties in setting of possibility theory. Besides, they emphasized using fuzzy set in scheduling problems, because fuzzy scheduling addressed not only scheduling under flexible constraints but also scheduling with incomplete or imprecise information. On the other hand, Gonzalez-Rodriguez et al. [35,36] argued that it was possible to model ill-known durations in job shop scheduling problems using fuzzy numbers instead of stochastic scheduling, because fuzzy theory and possibility theory could be considered as alternatives to the probabilistic models. Furthermore, applying fuzzy set has advantages over probability distributions in scheduling as fuzzy logic demands less data and thus, it reduces computational difficulties; also, it has expressive capability for uncertain events in order to handle incomplete knowledge of scheduling data. Moreover, Palacios et al. [37] declared that in the application of probability distributions, other representations of uncertain processing times would be a human-originated confidence interval, while we might have incomplete or little knowledge available for the Flexible Job Shop Problem (FJSP). In some studies on FJSP [38-41], both the processing time of operations on the eligible machine and completion time followed by 
the processing times are represented as triangular fuzzy numbers. The researchers have underlined using fuzzy data since processing times of machines are not precise enough. In fact, the problem is concerned with the scheduling scope and as in some real cases (especially in Iran), the database for the durations of surgeries is incomplete, modeling SCS with fuzzy data and finding an approach to tackling this problem would be very important.

This paper deals with a fuzzy surgical case scheduling problem in which all patients are elective and all processing time parameters are assumed fuzzy. We contribute to the field of operations research technique in employing available resources and making surgical case schedules by minimizing fuzzy makespan of operating theater under open scheduling policy. This paper has two novelties: a) extension of surgical case surgery problem using fuzzy processing time in all stages of operating room, and b) proposing and constructing a fuzzy ant system based on the model to tackle the problem under uncertain condition.

\section{Problem statement}

In this part, problem statement is thoroughly described. For this propose, details are divided in four subsections: a) fuzzy surgical case scheduling for elective patients, b) structure of no-wait multiresources fuzzy flexible job shop scheduling in operating theater, c) operations on triangular fuzzy numbers, and d) mathematical programming for fuzzy surgical case scheduling.

\subsection{Description of fuzzy surgical case scheduling for elective patients}

In this section, we give a brief description of surgical case processing from input to output in operating theater (Figure 1). First, the patient is transported from either ward or Ambulatory Surgical Unit (ASU) to PHU. While the patient is being held in PHU, the nurse checks the documents and prepares him/her for surgery. The patient occupies both nurse and PHU bed. Then, he/she is moved to operating room where the anesthetist manages anesthesia process and a specific surgeon performs surgical procedure on the case. In this stage, other resources such as nurse, OR, anesthetist, medical technicians, scrubs, and surgeon are allocated to the surgical case. In the end of the surgical process, anesthesia is reversed by the anesthetist and then, the patient is transported to PACU where he/she recovers from residual effects of anesthesia under the care of PACU nurse. In the third stage, the nurse and PACU bed are assigned to the patient. When effects of anesthesia are being diminished and the condition of the patient becomes stable, he/she is moved to several different destinations according to the existing conditions. The usual inpatient is returned to ward, the critical inpatient (e.g., cardiac or thoracic case) is moved directly to ICU, where he/she benefits from specially trained nurses and specialized equipment, and the outpatient is taken to ASU for going through a second recovery. It must be noted that all processing times including duration of pre-surgery stage, duration of surgery, and duration of post-surgery or recovery stage are under uncertain condition in operating theater and considered fuzzy based on information of the expert.

\subsection{Structure of no-wait multi-resources fFJSP in operating theater}

The various structures of the shop are taken into account to model and solve the SCS problem. For instance, Guinet and Chaabane [42] formulated a twostage hybrid flow shop model in order to minimize total overtime in operating rooms and Augusto et al. [43] modeled the SCS problem as a four-stage flow shop under open scheduling policy. In other studies [16,21,27], the similarities between operating room scheduling environment and Job Shop scheduling Problem (JSP) were observed. An FJSP was introduced by Pinedo [44]

\section{Operating Theater}

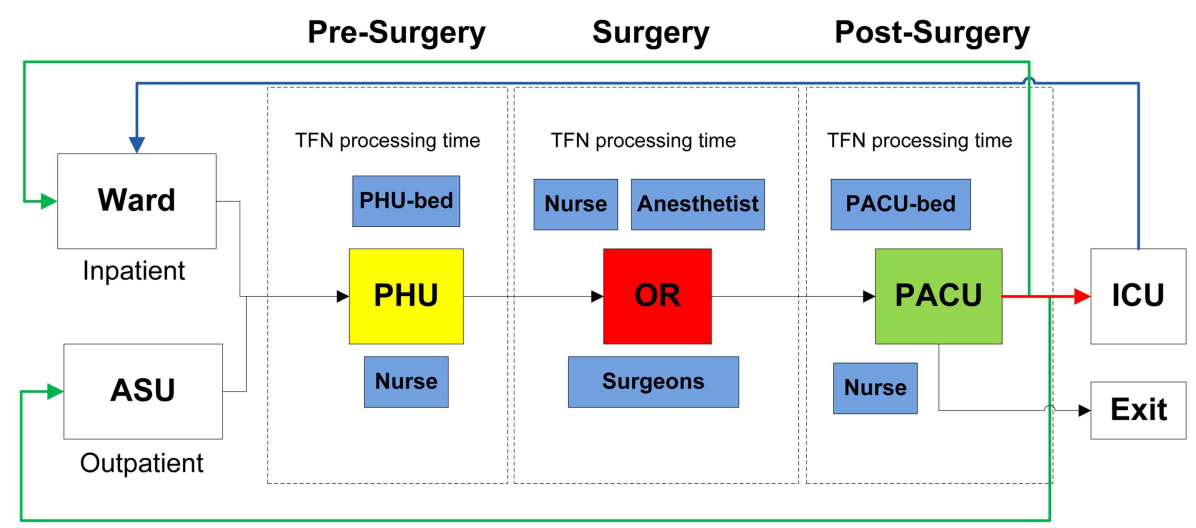

Figure 1. Patient flow in operating theater under uncertainty (blue-box shows resources). 


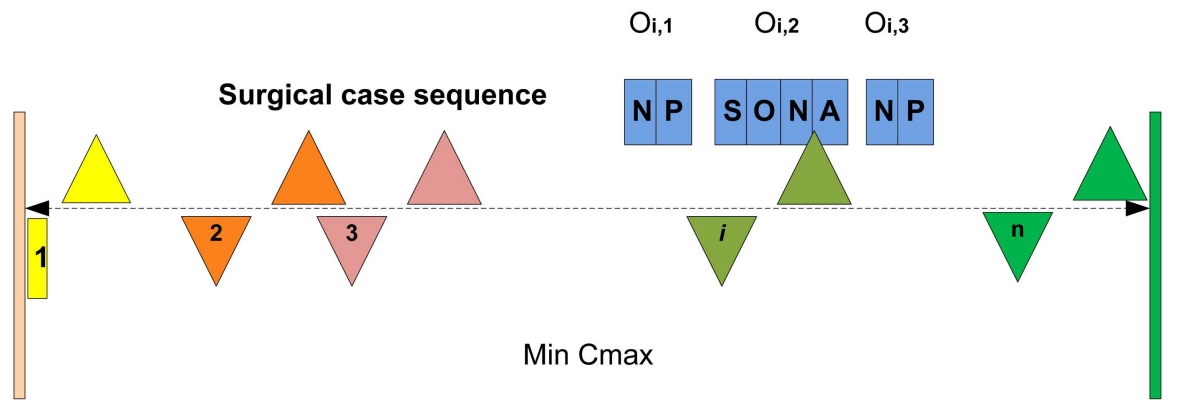

Figure 2. Fuzzy surgical case scheduling in operating theater.

as generalization of the job shop and the parallel machine environments. Each order has its own route to follow through the shop. Even though the flow shop may be modeled for operating theater in case of identical surgical procedures, in real world, various surgical cases with several surgical procedures require their own routes and this has motivated researchers to apply JSP environment in their studies. Pham and Klinkert [16] developed a novel multi-mode blocking JSP to model SCS problem in order to minimize makespan; however, Xiang et al. [21] considered generalization of job shop and then, formulated a multi-resource FJSP in order to minimize makespan by applying a novel two-level ACO procedure. The authors assumed that the operating sequence of three stages should be followed completely and one after another; therefore, this assumption made a constraint that followed the rules of no-wait flow shop. Pinedo [44] defined the no-wait requirement as a phenomenon that might occur in flow shops with zero intermediate storage. In no-wait situation, orders are not permitted to wait between two successive machines. By using this constraint in FJSP, the start time of the first stage in PHU for the surgical case has to be delayed to ensure that the case can go through the FJSP without having to wait for any resource. Therefore, the surgical cases are actually pulled down the line by the resources that have become idle. Xiang et al. [21] assumed that three general stages were essential for all cases in FJSP. We can observe flexibility in all stages because of diversity of resources in each stage. Furthermore, there are diverse routes for cases in the second stage because of the varieties of specific surgical procedure; this confirms the similarities between SCS in operating theater and FJSP environment. On the other hand, we describe fuzzy FJSP ( $f$ FJSP) in operating theater, because the fuzzy conditions are considered in the problem. There are some studies that have focused on $f$ FJSP in which the researchers have combined fuzzy scheduling and flexible scheduling in job shop environment. To solve an $f$ FJSP, a co-evolutionary genetic algorithm was proposed by Lei [38] as this problem had high complexity. The author introduced fuzzy Gantt chart and considered triangular fuzzy start time and completion time. In another work conducted by $\mathrm{Xu}$ et al. [39], a teaching-learning based optimization algorithm was introduced to tackle FJSP with fuzzy processing time. Liu et al. [40] proposed a fast estimation distribution algorithm to solve $f$ FJSP. Comparison of their approach with estimation distribution algorithm indicated that their algorithm outperformed the previous work. Also, Lin [41] applied a hybrid biogeographybased optimization as meta-heuristic to $f$ FJSP. In our study, we take into account the fuzzy flexible job shop environment introduced by Lei [38] for SCS problem because of similarities between fFJSP and SCS. As it is shown in Figure 2, there are a set of surgical cases, $S C=\left\{S C_{1}, S C_{2}, \ldots, S C_{n}\right\}$, to be operated by a combination of the available required resources, $R=\left\{\right.$ Nurse $_{1}, \ldots$, Nurse $_{m}$, Bed $_{1}, \ldots$, Bed $_{m}, A n_{1}$, $\left.\ldots, A n_{m}, S_{1}, \ldots, S_{m}, O R_{1}, \ldots, O R_{m}\right\}$, in each stage. Since there are three stages for each case, each surgical case, $S C_{i}$, is formed by a sequence of three operations, $\left\{O_{i, 1}, O_{i, 2}, O_{i, 3}\right\}$. Also, multiple resources assigned to the $i$-th surgical case for each stage are presented in blue boxes over the $S C_{i}$. The processing time of $O_{i j}$ (pre-surgery, surgery, post-surgery) for the required resources is represented as a Triangular Fuzzy Number $(\mathrm{TFN}), \tilde{P}_{i j r k}=\left(P_{i j r k}^{b}, P_{i j r k}^{m}, P_{i j r k}^{w}\right)$, where $P_{i j r k}^{b}$ pertains to the best processing time, $P_{i j r k}^{m}$ is the most probable processing time, and $P_{i j r k}^{w}$ relates to the worst processing time (Figure 3). Similarly, the fuzzy completion time of $O_{i j}$ (here notated by $E T$, which is the abbreviation of end time) is displayed as a TFN, $\tilde{E} T_{i j r k}=\left(E T_{i j r k}^{b}, E T_{i j r k}^{m}, E T_{i j r k}^{w}\right)$, where $E T_{i j r k}^{b}$ is

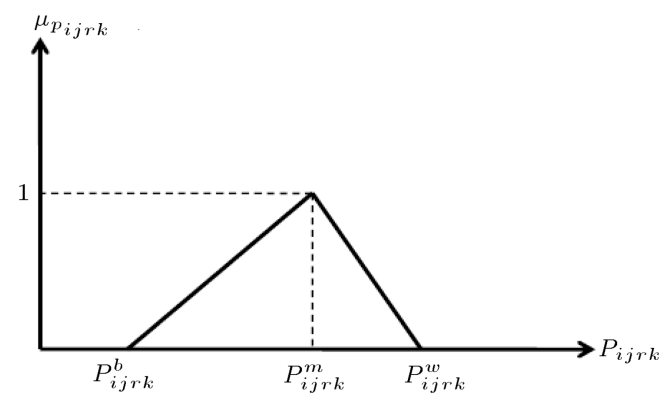

Figure 3. Triangular possibility distribution of fuzzy parameter $\left(P_{i j r k}\right)$. 
the best completion time, $E T_{i j r k}^{m}$ is the most probable completion time, and $E T_{i j r k}^{w}$ is the worst completion time. Hereafter, we call fuzzy Surgical Case Scheduling ( $f$ SCS) instead of $f$ FJSP problem, in which both allocation of efficient available resources and sequence of surgical cases for all resources are determined in order to minimize fuzzy makespan $\left(C_{\max }\right)$.

Based on the defined problem in this paper (extension of the model presented by Xiang et al. (2015) [21] to apply fuzzy duration time), the surgeon as the main resource plays the key role in operating room and is effective on duration of makespan as a key performance for assessing the scheduling problem. As it was explained, the OR scheduling problem is similar to FJSP environment, and the role of special surgeons in the operating room is similar to that of effective human resources in FJSP. In real cases, some assumptions may be relaxed to construct a complex and realistic model. For example, in real cases, the surgeon and her/his assistance are effective on makespan, simoltaneously, and a dual constraint model is required to consider this condition; this makes the model more complicated. However, in this study, a basic model is extended using fuzzy parameters to be practicable in some real cases.

\subsection{Operations on triangular fuzzy number}

For providing and using fuzzy duration time in operating room scheduling problem, the definitions of fuzzy number operations for TFN are essential to build a feasible schedule. The necessary operations in this paper include operation of adding two fuzzy numbers, the ranking approach to fuzzy numbers, and max operation of two fuzzy numbers. Summation operation is applied to add up the fuzzy start time and the process with fuzzy processing time, and to calculate the fuzzy completion time. The ranking operation is used to find the maximum fuzzy makespan value. The $\max$ operation is to determine the fuzzy start time of the process.

For two TFNs $\tilde{S}=\left(s_{1}, s_{2}, s_{3}\right)$ and $\tilde{P}=$ $\left(p_{1}, p_{2}, p_{3}\right)$, the summation is obtained by $\tilde{S}+\tilde{P}=$ $\left(s_{1}+p_{1}, s_{2}+p_{2}, s_{3}+p_{3}\right)$.

The following criteria are adopted to rank $\tilde{S}$ and $\tilde{P}$ in fuzzy scheduling [45]:

- Criterion 1: If $C_{1}(\tilde{S})=\frac{s_{1}+2 s_{2}+s_{3}}{4}>(<) C_{1}(\tilde{P})=$ $\frac{p_{1}+2 p_{2}+p_{3}}{4}$, then $\tilde{S}>(<) \tilde{P}$ is applied to rank them;

- Criterion 2: If both TFNs have identical values of $C_{1}$, then $C_{2}(\tilde{S})=s_{2}$ is compared with $C_{2}(\tilde{P})=p_{2}$ as the second criterion to rank them;

- Criterion 3: If both TFNs have the same $C_{1}$ and $C_{2}$, then the difference of the spreads, i.e., $C_{3}(\tilde{S})=$ $s_{3}-s_{1}$, is compared with $C_{3}(\tilde{P})=p_{3}-p_{1}$, is utilized as the last criterion to rank them.

Moreover, the approximate max of two TFNs $\tilde{S}$ and $\tilde{P}$ is obtained by the rule introduced in [38]. That is, if $\tilde{S}>\tilde{P}$, then $\tilde{S} \cup \tilde{P}=\tilde{S}$; else, $\tilde{S} \cup \tilde{P}=\tilde{P}$.

It should be notated that the above fuzzy equations are used to construct the schedule as applied before by Lei [38] and Lin [41] in order to solve $f$ FJSP. However, this is the first time that TFN processing times are provided in operating room scheduling, and fuzzy results of scheduling are presented according to fuzzy Gantt chart [38] as a robust output.

As it was noted in introduction, there are unpredicted incidents in operating room that increase usual surgery times and hence, some criteria such as makespan are increased. Thus, using deterministic times under uncertain condition does not give us a near optimal and reliable schedule. When there is incomplete or little knowledge available concerning surgery time in operating room, using TFNs according to the information of the expert in the model presents a robust schedule in which three makespans (the best, the most probable, and the worst) are obtained and the real makespan is obtained according to the knowledge of the expert considering the worst (under incidents that increase duration of surgery) or the best (surprising incidents that reduce duration).

\subsection{Mathematical programming based on no-wait multi-resource f FJSP}

Since the structure of $f$ FJSP is NP-hard problem, mathematical programming models cannot provide efficient tools to tackle the problems with large sizes, but they can be considered as the first step to develop an effective heuristic. In a study conducted by Demir and Isleyen [46], mathematical models for FJSP were evaluated. The models were divided into three classes based on their binary variables: sequence-position variable based model, precedence variable based model, and time-indexed model. The authors concluded that precedence variable based models, especially the MILP model developed by Ozguven et al. [47], outperformed other models with the lowest computation time. It is noteworthy that this model is the only linear one among them. Therefore, we formulate and develop the MILP model of Ozguven for $f$ SCS problem based on the no-wait multi-resource $f$ FJSP. Several assumptions are made in order to define daily $f$ SCS as follows:

1. Only elective patients (inpatients) are involved in this study and all of them are in access before scheduling on a given day at zero time; therefore, release/arrival time is assumed static and equal to zero;

2. Preemption is not allowed because no stages can be interrupted;

3. The required resources are determined before scheduling;

4. During working day and in zero time, all resources 
Table 1. Indices and sets for MILP model.

\begin{tabular}{cl}
\hline Index or set & \multicolumn{1}{c}{ Description } \\
\hline$I$ & Set of all the surgical cases \\
$S G$ & Set of specialties for surgeries \\
$I_{s}$ & Subset of surgical cases based of specialty of surgery $s$ \\
$J_{i}$ & Set of operations of case $i \in I$ \\
$R$ & Set of all resource types \\
$O_{i j}$ & Surgical case $i \in I$ in stage $j \in J$ \\
$R_{i j}$ & Set of capable resource types for operation $O_{i j}$ \\
& \\
$K_{r_{i j}}$ & Set of all the resources in resource type $r$ \\
& (with the exception of surgeon group) $r \in R-\{3\}, r_{i j} \in R_{i j}$ \\
$K_{r_{s}}$ & $s \in S G$ in resource type $r=3$ \\
\hline
\end{tabular}

Table 2. Parameters for MILP model.

\begin{tabular}{ll}
\hline \multicolumn{1}{c}{ Parameter } & \multicolumn{1}{c}{ Description } \\
\hline$\tilde{P}_{i j r k}=\left(P_{i j r k}^{b}, P_{i j r k}^{m}, P_{i j r k}^{w}\right)$ & $\begin{array}{l}\text { Fuzzy processing time (pre-surgery, surgery, and post-surgery } \\
\text { durations) of operation } O_{i j} \text { if performed with resource } k \text { of type } r\end{array}$ \\
$M$ & A large positive number \\
$n$ & Total number of surgical cases \\
$m_{r}$ & Total number of resources for each resource type $\left(8\right.$ types $\left.^{*}\right)$ \\
\hline
\end{tabular}

* In this study, 8 resource types are introduced.

are always available and there is no resource failure, so failure time is not assumed;

5. Clean-up and setup time are assumed to be included in surgery time and setup time is not sequence dependent;

6. All human and equipment resources are assumed to be identical in processing time with the exception of specific surgeons;

7. The priorities of all surgical cases are assumed to be identical;

8. The transportation times between operations (surgery stages) for each case are neglected, because the transporters are always assumed to be available;

9. All surgical cases must be operated in a sequence of three stages completely and one after another;

10. All data, consisting of pre-surgery, surgery, and post-surgery durations, are assumed uncertain or fuzzy in the problem;

11. Patients are only allowed to be operated by a subset of surgeons based on specialty.

Some assumptions in this paper are made to simplify the model under uncertainty. If some assumptions such as (5), (6), and (7) are relaxed, the complexity of the model and algorithm will increase. Therefore, Assumptions (5) and (7) are considered in MILP modeling based on $[2,21,27]$, and (6) is according to $[16,21]$. This paper focuses on the simple model with these assumptions under uncertain conditions. The model with these assumptions is solved by simulated data and suitable real cases in hospital. Some assumptions such as (1), (3), (4), (8), (9), and (11) are case dependent and a case is selected that is consistent with all assumptions for assessing the model. Finally, assumption (2) is consistent with real cases, because the surgical operating cannot be interrupted [19].

The sets/indices are described in Table 1 . In the problem, some elective patients in set $I$ and 8 resource types in set $R$, among which some types are involved in each surgical stage, are defined. In the first stage, there are two resource types including nurse and PHU bed and in the second stage, there are four types including surgeon, OR, nurse, and anesthesia.; finally, in the last stage, there are two types including PACU bed and nurse. This structure is based on [21]. Also, some parameters are defined in Table 2.

The applied variables in this mathematical model are divided into decision and auxiliary ones, which are described in the notation in Table 3 . In Table 3, aux- 
Table 3. Decision and auxiliary variables for MILP model.

\begin{tabular}{|c|c|}
\hline Variable & Description \\
\hline \multicolumn{2}{|l|}{ Decision variables } \\
\hline$S T_{i j r k}$ & The start time of operation $O_{i j}$ with resource $k$ of type $r$ \\
\hline$E T_{i j r k}$ & The end time of operation $O_{i j}$ with resource $k$ of type $r$ \\
\hline$C_{\max }$ & Makespan \\
\hline$v_{i j r k}$ & Equals 1 if operation $O_{i j}$ is performed on resource $k$ of type $r, 0$ otherwise \\
\hline$z_{i j h g r k}$ & Equals 1 if operation $O_{i j}$ precedes operation $O_{h g}$ on resource $k$ of type $r, 0$ otherwise \\
\hline$g_{i j r k}$ & Equals 1 if operation $O_{i j}$ is performed by surgeon $k$ of surgery specialty group $r=3$, \\
\hline & 0 otherwise. This variable is used for all resources involved in the second stage. \\
\hline \multicolumn{2}{|l|}{ Auxiliary variables } \\
\hline$E T_{i}$ & The completion time of surgical case $i$ \\
\hline
\end{tabular}

iliary variable $E T_{i}$ is employed to calculate makespan as the objective function.

A general model of MILP is formulated for the $f$ SCS problem with $n$ patients as follows:

$\min C_{\max }$,

s.t.

$$
\begin{aligned}
& E T_{i} \leq C_{\max } \forall i \in I, \\
& E T_{i} \geq \sum_{k \in K_{r_{i j}}} E T_{i j r k} \forall i \in I, \quad j=3, \quad r=8, \\
& S T_{i j r k}+E T_{i j r k} \leq M v_{i j r k} \forall i \in I / I_{s}, \\
& j \in\{1,2,3\}, \quad r \in R_{i j}\{1,2,3,7,8\}, \quad k \in K_{r_{i j}}, \quad(4) \\
& S T_{i j r k}+\tilde{P}_{i j r k}-M\left(1-v_{i j r k}\right) \leq E T_{i j r k} \forall i \in I / I_{s}, \\
& \quad j \in\{1,2,3\}, \quad r \in R_{i j}\{1,2,3,7,8\}, \quad k \in K_{r_{i j}},
\end{aligned}
$$

$$
S T_{i j r k}+E T_{i j r k} \leq M v_{i j r k} \forall i \in I, j=2,
$$$$
r \in R_{i j}\{4,5,6\}, \quad k \in K_{r_{i j}},
$$$$
S T_{i j r k}+\sum_{k \in K_{r_{s}}} \tilde{P}_{i j r k} g_{i j r k}-M\left(1-v_{i j r k}\right)
$$$$
\leq E T_{i j r k} \forall i \in I \text {, }
$$$$
j=2, \quad r \in R_{i j}\{4,5,6\}, \quad k \in K_{r_{i j}},
$$

$E T_{h g r k}-M z_{i j h g r k} \leq S T_{i j r k} \forall i, \quad h \in I / I_{s}, \quad i \ll h, j$,

$g \in J, \quad r \in R_{i j} \cap R_{h g}, \quad k \in K_{r_{i j}} \cap K_{r_{h g}}$,

$$
\begin{aligned}
& \sum_{k \in K_{r_{i j}}} S T_{i j r k}=\sum_{k \in K_{r_{i(j-1)}}} E T_{i(j-1) r k} \forall i \in I / I_{s}, \\
& j \in\{2,3\}, \quad r \in R_{i j}, \\
& \sum_{k \in K_{r_{i j}}} S T_{i j r k}=\sum_{k^{\prime} \in K_{r_{i j}}} S T_{i j r^{\prime} k^{\prime}} \forall i \in I / I_{s}, \\
& j \in J, r, r^{\prime} \in R_{i j}, \\
& \sum_{k \in K_{r_{i j}}} E T_{i j r k}=\sum_{k^{\prime} \in K_{r_{i j}}} E T_{i j r^{\prime} k^{\prime}} \forall i \in I / I_{s}, \\
& j \in J, r, \quad r^{\prime} \in R_{i j},
\end{aligned}
$$

$\sum_{k \in K_{r_{s}}} g_{i j r k}=1 \forall i \in I_{s}, \quad j=2, \quad r=3$,

$S T_{i j r k} \geq 0 \forall i \in I, \quad j \in J, \quad r \in R_{i j}, \quad k \in K_{r_{i j}}$,

$E T_{i j r k} \geq 0 \forall i \in I, \quad j \in J, \quad r \in R_{i j}, \quad k \in K_{r_{i j}}$,

$E T_{i} \geq 0 \forall i \in I$,

$C_{\max } \geq 0$ 


$$
\begin{gathered}
v_{i j r k} \in\{0,1\} \forall i \in I, \quad j \in J, \quad r \in R_{i j}, \quad k \in K_{r_{i j}}, \\
g_{i j r k} \in\{0,1\} \forall i \in I_{s}, \quad j=2, \quad r=3, \quad k \in K_{r_{s}}, \\
z_{i j h g r k} \in\{0,1\} \forall i, \quad h \in I / I_{s}, \quad i \ll h, j, \quad g \in J, \\
r \in R_{i j} \cap R_{h g}, \quad k \in K_{r_{i j}} \cap K_{r_{h g} .}
\end{gathered}
$$

In the above model, Eq. (1) states minimum objective functions, i.e., makespan. Constraint (2) determines makespan based on completion time of surgical cases. Eq. (3) reflects completion times of surgical cases at the end of the third stage. Constraints Eqs. (4) and (5) make sure that the difference between the start time and the end time of the operation of the surgical cases in the first to third stages (only surgeon in the second stage) is equal to the processing time of these stages for related resources. Constraints (6) and (7) guarantee the same requirements for equations (4) and (5); however, among the other involved resources in the second stage, surgeon is an exception, i.e., (resource types \#4, \#5, and \#6). Constraints (8) and (9) are used to specify that two different operations of $O_{i j}$ and $O_{h g}$ cannot be performed at the same time on any resource in the set $R_{i j} \cap R_{h g}$. Eq. (10) ensures that the $j$ th operation of a surgical case should be started exactly after the completion time of the $(j-1)$ th operation of the same surgical case. Constraints (11) and (12) specify that all the required resources for each surgery stage should have the same start time and completion time, respectively. Eq. (13) demands that one and only one resource from each resource type can be allocated to an operation of surgical case. Finally, Constraint (14) states that one and only one surgeon from surgery specialty group can perform the surgical procedure and it is possible that others are idle or allocated to other cases. Constraints (15)-(21) are positive and binary decision variables.

Since our mathematical programming represents a possibilistic model, first, we transform it into a crisp equivalent model based on the Weighted Average Method (WAM) introduced by Noori-Darvish [48]. We consider WAM for defuzzification of processing time in left-hand side of Constraints (5) and (7) as follows:

$$
\begin{aligned}
S T_{i j r k} & +\left(w_{1} P_{i j r k}^{b}+w_{2} P_{i j r k}^{m}+w_{3} P_{i j r k}^{w}\right) \\
& -M\left(1-v_{i j r k}\right)=E T_{i j r k} \forall i \in I / I_{s}, \\
& j \in\{1,2,3\}, \quad r \in R_{i j}\{1,2,3,7,8\}, \quad k \in K_{r_{i j}},
\end{aligned}
$$

$$
S T_{i j r k}+\sum_{k \in K_{r s}}\left(w_{1} P_{i j r k}^{b}+w_{2} P_{i j r k}^{m}+w_{3} P_{i j r k}^{w}\right) g_{i j r k}
$$

$$
\begin{aligned}
& -M\left(1-v_{i j r k}\right)=E T_{i j r k} \forall i \in I, \\
& j=2, \quad r \in R_{i j}\{4,5,6\}, \quad k \in K_{r_{i j}} .
\end{aligned}
$$

$\mathrm{Xu}$ et al. [39] defined $\left(x_{1}+2 x_{2}+x_{3}\right) / 4$ as Average Response Variable value (ARV) for defuzzification of TFN, $X=\left(x_{1}, x_{2}, x_{3}\right)$. We set $w_{1}=0.25, w_{2}=0.5$, $w_{3}=0.25$ for processing time. All positive decision variables such as start time, end time, and makespan are obtained as crisp numbers.

In our model, the start and end times of each surgery, the start and end times for the involved resources in each stage of surgery, and the required resources in each stage of surgery are variable in order to minimize makespan. Also, some parameters are needed as input to the model, such as available resources in each type, fuzzy processing time of each stage for each surgical case, specialist surgeon groups, and surgeons inside each specialist group. Finally, the output of the model includes variables representing the start and end times of surgeries to determine sequencing surgical cases as a part of the problem, and the start and end times of involved resources to determine the resource assigned to each surgical case as another part of the problem.

\section{Fuzzy ant system for solving $f$ SCS}

In this paper, we suggest a meta-heuristic approach in order to tackle the combinatorial nature of $f$ SCS problem. In many researches in the field of crisp SCS problem, some heuristic or meta-heuristic procedures such as genetic algorithm [12,13], column generation based heuristic [10], tabu search [31,32], and ant colony optimization [21] have been developed to achieve near optimal solutions. As it was described, structure of the problem is similar to that of $f \mathrm{FJSP}$ and thereby, Ant System (AS) algorithm is extended to solve optimization problems such as fFJSP. [21] proposed an ACO algorithm with two-level hierarchical graph (outer and inner graphs) to solve SCS. The authors considered outer and inner graphs in order to combine sequencing surgical cases and allocating resources. The first ACO algorithm, namely AS, was introduced by Dorigo et al. [49] as an optimizer, learning, and natural algorithm; also, it was applied to tackle Traveling Salesman Problem (TSP) by Dorigo et al. [50]. To the best of our knowledge, in the area of $f \mathrm{SCS}$ in the literature, no research is available that employs AS algorithm; therefore, we develop it for operating theater problem with fuzzy parameters. Then, we compare our proposed procedure with the surgical case scheduling solved by using FCFS rule and efficiency of the method is determined based on quality of the solutions obtained. Our elementary AS algorithm is based upon the proposed algorithm by Xiang et al. [21] 
and we extend it so as to solve $f$ SCS.

\subsection{Description of fuzzy AS algorithm}

Two-level ACO algorithm is tailored by mapping cities to surgical cases and thereby, a nodes tour turns to be the sequence of surgical cases. In Xiang's procedure, surgical cases are sequenced in the outer graph and the required multi-resource types of every stage are allocated to surgical cases in the inner graph. Available resources of the same resource type are represented by nodes inside the inner graph. The resource assigned to the surgical case in each stage is determined based on the path the ant forages in the inner graph. A mix pheromone update strategy is defined for the algorithm and it comprises one local and two global strategies. In the outer level, the best ant updates the trails based on the global iteration best strategy in order to search for the best sequence. In the inner level, surgeryrelated pheromone is defined to save the information that connects the surgical case to the required resource based on global strategy, while an inner resourcerelated pheromone is defined to record the information related to resource utilization based on local strategy. It must be noted that local updating is effective until the ant forages the path of the inner graph and it is invalid after going out. Algorithm 1 shows the framework of fuzzy ant system.

It must be noted that the start (available) time of all resources before planning in the algorithm is set to $(0,0,0)$.

The procedure of pheromone updates for the algorithm fuzzy AS ( $f$ AS) and transition rules are described in this section. Since the objective value in our problem is fuzzy makespan, we employ the same defuzzification method used in MILP to transform the fuzzy $\tilde{C}_{\max }$ into a crisp $C_{\max }$. This approach is applied in order to calculate crisp $\Delta \tau_{i j}^{k}$ in Eq. (25) as Kuo et al. [51] discussed in their fuzzy ACO method. In $f \mathrm{AS}$ algorithm, all computations are based on operations of triangular fuzzy numbers while pheromone update computation is based on ordinary deterministic operations. Pheromone update strategy in outer graph is based on the following equation:

$$
\tau_{i j}(t+1)=(1-\rho) \cdot \tau_{i j}(t)+\sum_{k=1}^{m} \Delta \tau_{i j}^{k}
$$

where:

$$
\Delta \tau_{i j}^{k}= \begin{cases}\frac{Q}{C_{\max }} & \text { if ant } k \text { goes through }(i, j) \\ 0, & \text { in this iteration } \\ 0 \text { otherwise }\end{cases}
$$

and $\Delta \tau_{i j}^{k}$ is increasing value of pheromone from patient $i$ to patient $j$ in the iteration related to the route of the $k$ th ant and crisp $C_{\max }$ is makespan of the $k$ th agent. Besides, Pheromone update strategy in the inner graph is based on the following equation:

$$
i n\left(\tau_{t m}^{i}(t+1)\right)=(1-\rho) \cdot i n\left(\tau_{t m}^{i}(t)\right)+\sum_{k=1}^{m} \Delta i n\left(\tau_{t m}^{i, k}\right),
$$

where:

$$
\Delta i n\left(\tau_{t m}^{i b e s t}\right)= \begin{cases}\frac{Q}{C_{\max }} & \begin{array}{l}
\text { if ant } k \text { goes through surgery } \\
(i) \text { with resource graph }(t, m)
\end{array} \\
0, & \text { otherwise }\end{cases}
$$

and $\operatorname{\Delta in}\left(\tau_{t m}^{i, k}\right)$ is increasing value of pheromone for

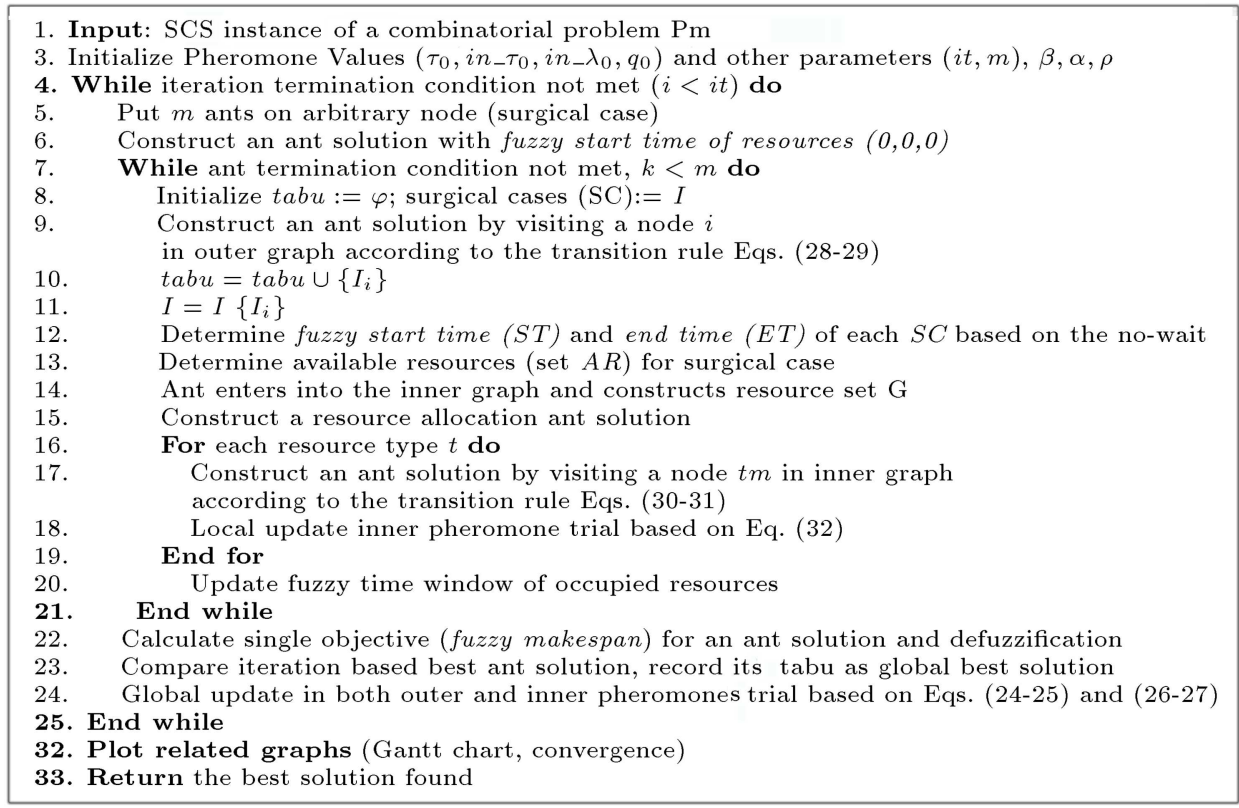

Algorithm 1. Two-level fuzzy ant system. 
patient $i$ by choosing resource $m$ from resource type $t$ in the iteration related to the route of the $k$ th ant and crisp $C_{\max }$ is makespan of the $k$ th agent.

Transition rules in the outer and inner graphs are shown in the following equations. In the outer graph, choosing the probability of patient $j$ after patient $i$ is presented as follows:

$$
P_{i j}^{k}(t)=\frac{\left[\tau_{i j}(t)\right]^{\alpha} \cdot\left[\eta_{i j}\right]^{\beta}}{\sum_{l \in I_{i}^{k}}\left[\tau_{i l}(t)\right]^{\alpha} \cdot\left[\eta_{i l}\right]^{\beta}} \quad \text { if } j \in I_{i}^{k},
$$

where $\tau_{i j}(t)$ is pheromone value of the current iteration for arc patients $i$ to $j$, and $\alpha$ and $\beta$ are pheromone factor and heuristic factor, respectively. Heuristic information of the problem in the outer graph for arc patients $i$ to $j$ is $\eta_{i j}$, which is presented based on Xiang's equation as follows:

$$
\begin{aligned}
\eta_{i j}= & \left(T_{j 1}+T_{j 3}+\max \left(T_{j 2}^{S G m}\right)\right) / \\
& \left(T_{j 1}+T_{j 3}+\max \left(T_{j 2}^{S G m}\right)+A\right),
\end{aligned}
$$

where, $T_{j 1}, T_{j 2}^{S G m}$, and $T_{j 3}$ are crisp processing times (pre-surgery, surgery, and post-surgery) defuzzified based on Xu's equation; also, parameter $A$ is constant and determined based on the level of initial pheromone. In the inner graph, choosing probability of resource $m$ from resource type $t$ is shown as follows:

$$
\begin{aligned}
& P_{t m}^{k i}(t)=\frac{\left[i n\left(\tau_{t m}^{i}(t)\right) \cdot i n\left(\lambda_{m}\right)\right]^{\alpha} \cdot\left[i n\left(\eta_{t m}\right)\right]^{\beta}}{\sum_{g \in G_{i}^{k}}\left[i n\left(\tau_{t g}^{i}(t)\right) \cdot i n\left(\lambda_{g}\right)\right]^{\alpha} \cdot\left[i n\left(\eta_{t g}\right)\right]^{\beta}} \\
& \text { if } j \in G_{i}^{k},
\end{aligned}
$$

where, $i n\left(\tau_{t m}^{i}(t)\right)$ is pheromone value of the current iteration for edge resource $m$ from resource type $t$, and $i n\left(\eta_{t m}\right)$ is heuristic information in the inner graph for edge resource $m$ from resource type $t$, which is presented as follows:

$$
i n\left(\eta_{t m}\right)=B /\left(E S_{i l}^{t m}+T_{i l}^{t m}\right)
$$

where, $E S_{i l}^{t m}$ is the crisp earliest time for the available resource $m$ from resource type $t$ for patient $i$ in stage $l$, and $T_{i l}^{t m}$ is crisp processing time of patient $i$ in stage $l$ when resource $m$ from resource type $t$ is involved. These parameters are defuzzified based on $\mathrm{Xu}$ 's equation. Also, parameter $B$ is constant and determined based on the level of initial pheromone. On the other hand, in $\left(\lambda_{t m}\right)$ is resource-related pheromone in the inner graph, which is described in Eq. (32). Finally, there is a new pheromone update strategy, namely resource-related pheromone in $\left(\lambda_{t m}\right)$, which is shown in the array of Figure 4 ; it is very effective for resource utilization (red boxes are less probable to be selected by ant in the inner graph). Because this

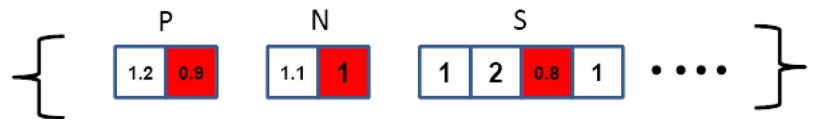

Figure 4. The sample of inner resource related pheromone for the resources of the operating room.

strategy decreases pheromone value of each resource node in the inner graph, selection probability of the same resource by the ant is diminished and other resources can have the opportunity to be selected. This strategy is performed locally. When an agent trails pheromone in the inner graph and selects a resource for a case in each stage, the value of the resourcerelated pheromone is updated and then, it will be reset to the preliminary pheromone $\left(i n\left(\lambda_{0}\right)\right)$ after obtaining a feasible sequence by the agent.

$$
\text { in }\left(\lambda_{t m}\right)=i n\left(\lambda_{t m}\right)-q_{0},
$$

where, $q_{0}$ states decremented pheromone value.

\section{Computational experiments}

\subsection{Illustrative examples}

In order to evaluate the proposed approaches, we consider three surgery test cases. These cases are classified into small, medium, and large as they are different in terms of duration of surgery, the number of the surgery cases, and the allocated resources. It should be noted that each case consists of three various instances or problems. Categories of cases and their specifications are shown in Table 4. Surgeries are categorized into five types of small, medium, large, extra-large, and special based on their durations (Table 5); deterministic durations of surgeries are determined based on the simulation model constructed by Xiang et al. [21] and each problem in each case can be generated based on the specific structure of the duration type of the surgery. Since processing durations in this paper are fuzzy numbers, we provide fuzzy processing times $(t-u, t, t+v)$ in which $u$ and $v$ are approximated between intervals of $1 \%$ to $30 \%$ of deterministic duration $(t)$, randomly. As it is observed in Table 4, three problems for each case are different in terms of size of surgery (column 3 ), size of resources (column 4-9), and surgery type structure (column 10). The $f$ AS algorithm was coded in MATLAB language run on an Intel Core (TM) Duo CPU T2450, 2.00 GHz computer with $1 \mathrm{~GB}$ of RAM. Moreover, MILP model was coded in GAMS software and run by CPLEX solver.

\subsection{Setting parameters of the proposed algorithm}

Various parameters in general ACO algorithms are effective on their performance, especially solution quality and computational time. For instance, some 
Table 4. Test cases and structures.

\begin{tabular}{|c|c|c|c|c|c|c|c|c|c|}
\hline Case & Problem & $\begin{array}{c}\text { Surgical } \\
\text { case }\end{array}$ & PHU bed & Nurses & Surgeons & ORs & $\begin{array}{c}\text { PACU } \\
\text { beds }\end{array}$ & Anesthesia & $\begin{array}{l}\text { Surgery type } \\
\text { (S:M:L:EL:S) }\end{array}$ \\
\hline \multirow{3}{*}{ Case 1} & 1 & 8 & 1 & 5 & 5 & 2 & 2 & 5 & $2: 4: 1: 1: 0$ \\
\hline & 2 & 10 & 2 & 8 & 6 & 4 & 4 & 6 & $2: 6: 1: 1: 0$ \\
\hline & 3 & 10 & 2 & 8 & 6 & 4 & 4 & 6 & $2: 5: 2: 1: 0$ \\
\hline \multirow{3}{*}{ Case 2} & 1 & 15 & 3 & 10 & 6 & 4 & 3 & 8 & $3: 9: 2: 1: 0$ \\
\hline & 2 & 20 & 3 & 15 & 10 & 5 & 4 & 8 & $4: 12: 3: 1: 0$ \\
\hline & 3 & 20 & 3 & 15 & 10 & 5 & 4 & 8 & $4: 10: 3: 3: 0$ \\
\hline \multirow{3}{*}{ Case 3} & 1 & 30 & 4 & 19 & 10 & 6 & 5 & 9 & $7: 16: 3: 2: 2$ \\
\hline & 2 & 30 & 4 & 22 & 12 & 6 & 5 & 11 & $5: 15: 3: 4: 3$ \\
\hline & 3 & 30 & 5 & 22 & 12 & 6 & 6 & 12 & $3: 15: 3: 4: 5$ \\
\hline
\end{tabular}

Table 5. Duration of surgery stages in different surgery types.

\begin{tabular}{cccccccc}
\hline & & \multicolumn{5}{c}{ Surgery case } & \\
\cline { 3 - 7 } & Pre-surgery & Small & Medium & Large & E-large & Special & Post-surgery \\
\hline \multirow{2}{*}{ Duration } & Random. & Random. & Random. & Random. & Random. Random. & Random. \\
& normal & normal & normal & normal & normal & normal & normal \\
$(\min )$ & $(8,2)$ & $(33,15)$ & $(86,17)$ & $(153,17)$ & $(213,17)$ & $(316,62)$ & $(28,17)$ \\
\hline
\end{tabular}

Table 6. Setting parameters of AS algorithm.

\begin{tabular}{ccccccc}
\hline Case no. & $($ Max-it, $\mathbf{m})$ & $\boldsymbol{q}_{\mathbf{0}}$ & $\boldsymbol{\lambda}_{\mathbf{0}}$ & $\boldsymbol{\alpha}$ & $\boldsymbol{\beta}$ & $\boldsymbol{\rho}$ \\
\hline 1 & $25-40$ & 0.1 & 4 & 0.9 & 5 & 0.1 \\
2 & $30-40$ & 0.1 & 5 & 0.9 & 12 & 0.1 \\
3 & $60-50$ & 45 & 9 & 0.9 & 2 & 0.2 \\
\hline
\end{tabular}

parameters like the number of ants $(m)$, the number of iterations $(\operatorname{Max}-I t)$, evaporation rate $(\rho)$, weighted importance of pheromone $(\alpha)$, and weighted importance of heuristic information $(\beta)$ are considered as the elementary ones. However, two novel parameters are introduced based on Xiang's two-level ACO, which are inner resource-related pheromone $\left(\lambda_{0}\right)$ and decremented pheromone value $\left(q_{0}\right)$. We design experiments based on orthogonal Taguchi Design Of Experiment (DOE) in order to examine the effect of parameter settings on each ARV obtained by the algorithm for the three test case groups. The final parameter settings of the algorithm for three cases are displayed in Table 6 .

\subsection{Evaluation of the performance of the algorithm for all the considered instances}

Assessing the proposed algorithm is done in this subsection in two parts; firstly, the algorithm is validated for the small simulated case in comparison with MILP model and after that, it is evaluated for small to large simulated cases in comparison with FCFS rule. Secondly, a real case in accordance with our model (considering all assumptions) in a private hospital in Isfahan province is presented to evaluate the proposed algorithm against real data.

Firstly, we ran fFCFS method and MILP model for the very small case as presented in Table 7 along with small data of Table 4 to validate the approach. The algorithm was repeated 10 times and the makespan mean found by $f$ AS was compared with that by MILP. It is noteworthy that $f \mathrm{AS}$ algorithm was validated in comparison with MILP model for five small cases as shown in Table 8 in which columns 2 and 3 show the results of the methods and columns 4 and 5 display the

Table 7. Test cases for comparing MILP and fuzzy ACO algorithm.

\begin{tabular}{ccccccccc}
\hline Sample & $\begin{array}{c}\text { Surgical } \\
\text { case }\end{array}$ & PHU bed & Nurses & Surgeons & ORs & $\begin{array}{c}\text { PACU } \\
\text { beds }\end{array}$ & Anesthesia & $\begin{array}{c}\text { Surgery type } \\
\text { (S:M:L:EL:S) }\end{array}$ \\
\hline 1 & 3 & 2 & 6 & 6 & 2 & 2 & 2 & $0: 2: 1: 0: 0$ \\
2 & 5 & 1 & 9 & 6 & 6 & 2 & 6 & $2: 2: 1: 0: 0$ \\
\hline
\end{tabular}


Table 8. Comparison of the performances of the fuzzy AS and MILP.

\begin{tabular}{lcccc}
\hline Sample no. & MILP & $\boldsymbol{f A S}$ & GAP $(\boldsymbol{\%})$ & CT(MILP $/ \boldsymbol{f A S})$ \\
\hline 1 (very small) & $\mathbf{1 6 0 . 2 5}$ & $\mathbf{( 1 4 4 , \mathbf { 1 6 0 , 1 7 7 } ) = \mathbf { 1 6 0 . 2 5 }}$ & $0.00 \%$ & $8 / 15$ \\
2 (very small) & $\mathbf{2 4 9 . 5}$ & $\mathbf{( 2 3 4 , 2 5 0 , 2 6 4 )}=\mathbf{2 4 9 . 5}$ & $0.00 \%$ & $180 / 25$ \\
3 (small) & $\mathbf{4 2 1 . 7 5}$ & $\mathbf{( 3 9 1 , 4 3 7 , 4 8 4 )}=\mathbf{4 3 7 . 2 5}$ & $3.67 \%$ & $1000 / 50$ \\
4 (small) & $\mathbf{2 7 0 . 5}$ & $\mathbf{( 2 4 8 , 2 9 3 , 3 3 6 )}=\mathbf{2 9 2 . 5}$ & $8.13 \%$ & $1200 / 80$ \\
5 (small) & $\mathbf{2 8 3 . 7 5}$ & $\mathbf{( 2 5 3 , 2 9 7 , 3 4 7 )}=\mathbf{2 9 8 . 5}$ & $5.19 \%$ & $1200 / 80$ \\
6 (medium) & - & $\mathbf{( 3 3 6 , 3 8 1 , 4 3 3 )}=\mathbf{3 8 2 . 7 5}$ & - & $-/ 120$ \\
\hline
\end{tabular}

Table 9. Comparison of the performances of the algorithms in all the considered test case problems.

\begin{tabular}{|c|c|c|c|c|}
\hline \multirow[t]{2}{*}{ Problems } & \multirow[t]{2}{*}{ Method } & \multicolumn{3}{|c|}{ Makespan } \\
\hline & & Average & Best & Worst \\
\hline \multirow[t]{2}{*}{ Instance 1} & $f \mathrm{AS}$ & $(397.8,443.6,492.4)$ & $(391,437,484)$ & $(404,451,501)$ \\
\hline & $f \mathrm{FCFS}$ & $(500,547,599)$ & $(500,547,599)$ & $(500,547,599)$ \\
\hline \multirow[t]{2}{*}{ Instance 2} & $f \mathrm{AS}$ & $(247.6,299.8,353.7)$ & $(248,293,336)$ & $(220,310,401)$ \\
\hline & $f \mathrm{FCFS}$ & $(328,389,450)$ & $(328,389,450)$ & $(328,389,450)$ \\
\hline \multirow[t]{2}{*}{ Instance 3} & $f \mathrm{AS}$ & $(253.1,302.2,356.7)$ & $(253,297,347)$ & $(284,312,339)$ \\
\hline & $f \mathrm{FCFS}$ & $(359,415,479)$ & $(359,415,479)$ & $(359,415,479)$ \\
\hline \multirow[t]{2}{*}{ Instance 4} & $f \mathrm{AS}$ & $(339.1,388.3,438)$ & $(336,381,433)$ & $(334,394,448)$ \\
\hline & $f \mathrm{FCFS}$ & $(458,496,542)$ & $(458,496,542)$ & $(458,496,542)$ \\
\hline \multirow[t]{2}{*}{ Instance 5} & $f \mathrm{AS}$ & $(359.6,403.2,449.3)$ & $(358,398,444)$ & $(365,406,454)$ \\
\hline & $f \mathrm{FCFS}$ & $(558,603,651)$ & $(558,603,651)$ & $(558,603,651)$ \\
\hline \multirow[t]{2}{*}{ Instance 6} & $f \mathrm{AS}$ & $(419.2,470.1,528.3)$ & $(417,468,520)$ & $(407,476,555)$ \\
\hline & $f \mathrm{FCFS}$ & $(615,693,790)$ & $(615,693,790)$ & $(615,693,790)$ \\
\hline \multirow[t]{2}{*}{ Instance 7} & $f \mathrm{AS}$ & $(502.4,577.3,652.6)$ & $(485,570,653)$ & $(532,583,633)$ \\
\hline & $f \mathrm{FCFS}$ & $(703,770,834)$ & $(703,770,834)$ & $(703,770,834)$ \\
\hline \multirow[t]{2}{*}{ Instance 8} & $f \mathrm{AS}$ & $(614.2,679.7,746.1)$ & $(630,676,722)$ & $(605,684,764)$ \\
\hline & $f \mathrm{FCFS}$ & $(741,832,923)$ & $(741,832,923)$ & $(741,832,923)$ \\
\hline \multirow[t]{2}{*}{ Instance 9} & $f \mathrm{AS}$ & $(727.7,801.5,890)$ & $(737,799,873)$ & $(724,801,909)$ \\
\hline & $f \mathrm{FCFS}$ & $(815,907,1012)$ & $(815,907,1012)$ & $(815,907,1012)$ \\
\hline
\end{tabular}

Table 10. ANOVA results for ARV (crisp makespan).

\begin{tabular}{lccccc}
\hline Source of variation & DF & SS & MS & F & $P$-value \\
\hline Method & 1 & 924787 & 924787 & 130247.40 & 0.000 \\
Sample problem & 8 & 4951180 & 618897 & 87165.82 & 0.000 \\
Interaction & 8 & 102208 & 12776 & 1799.37 & 0.000 \\
Error & 162 & 1150 & 7 & & \\
Total & 179 & 5979324 & & & \\
\hline
\end{tabular}

gaps between results of the two methods and between their computational times.

After determination of the best parameter setting for solving the small to large instances tested, we ran $f \mathrm{AS}$ algorithm in order to evaluate the performance of the algorithm. Table 9 presents the comparison between the average performance of $f \mathrm{AS}$ and the results of $f$ FCFS for all instances. The first and second columns of Table 9 display the considered test problems and approaches, respectively. The next three columns represent the average, best, and worst fuzzy makespans for the solutions obtained. As the table shows, $f \mathrm{AS}$ outperforms $f$ FCFS in solving all instances from small to large sizes.

The ANOVA test results for ARV as a response variable are presented in Table 10. Considering the $P$-values for the main effects of the sample problem and the algorithm, it is seen that the effect of 


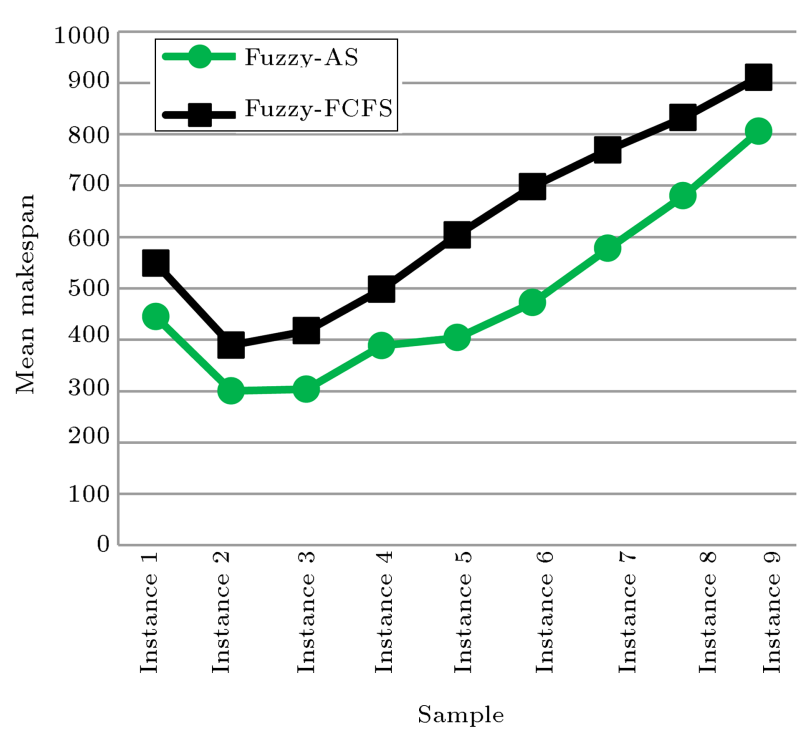

Figure 5. Interaction between the algorithms and test problems for the ARV (crisp makespan).

the aforementioned factor is significant. It means that there is significant difference between the mean makespans obtained for the two methods as well as between those obtained for the nine sample problems. Moreover, interaction effect of problems and algorithms is significant. Figure 5 shows that the proposed $f$ AS algorithm obtains lower ARV than fFCFS does for each instance.

A hospital from Isfahan, Iran, is selected, which is consistent with all assumptions of the model under open strategy. For instance, in this real case of operating theatre, priorities of patients are assumed identical in some days and are not important in planning management, because surgical cases are categorized in the same age group and there are not cardiac, thoracic, and some urgent cases. To implement the proposed algorithm for the real case, the specialist surgeons are divided into two groups for inpatients and outpatients. The specialist group for inpatients consists in only a surgeon assigned to a surgical case and this is a clinical decision, whereas the group for outpatients includes at least two general surgeons with different processing times assigned to the case; this is not necessarily a clinical decision, because there are more resources to be allocated. Therefore, assigning a general surgeon by the model can optimize makespan and improve the schedule.

Real data, including fuzzy durations for all stages of each surgical case, were collected in 8 different days by getting data from experts such as anesthetists, nurses, and surgeons. Therefore, each instance included elective cases to be operated during a day, fuzzy durations for cases, and available required resources for all surgeries in the same day. The proposed algorithm was repeated 10 times and the mean makespan

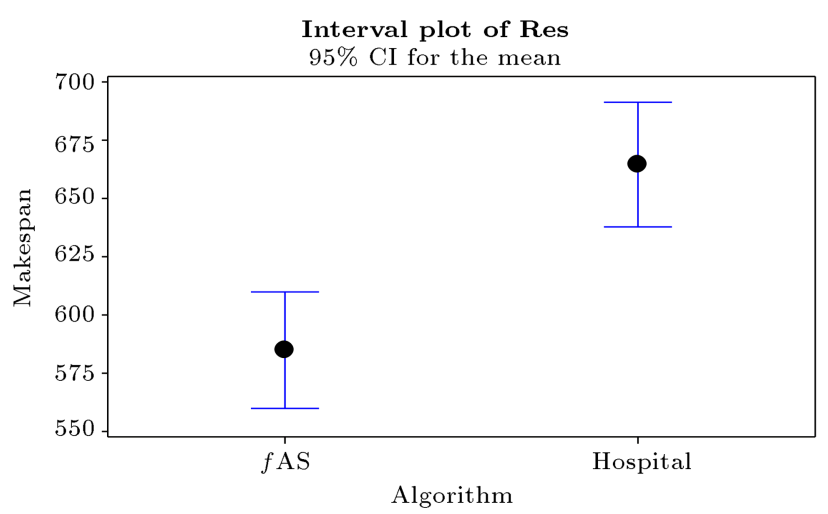

Figure 6. Main effect plot and LSD intervals (at 95\% confidence level) of makespan for algorithms.

achieved by $f$ AS was compared with the result of the real hospital planning. The global solutions achieved by $f$ AS for all cases tested outperformed real planning as shown in Table 11. Also, the ANOVA test results for the achieved ARV (algorithm and real planning) methods are displayed in Table 12. Considering the results, the $P$-value for the main effect of the solution method is less than 0.05. So, the main effect of the solution method is significant for the mean makespans. It means that there is significant difference between the mean makespans obtained for the proposed algorithm and real planning. Moreover, Figure 6 shows the interval plots (at 95\% confidence level) of makespan for the methods, indicating that the proposed $f$ AS algorithm obtains lower ARV than the real planning does for all instances.

\section{Conclusion}

In this paper, we addressed the surgical case scheduling problem in operating theater under uncertain condition of service time; a new approach was proposed so as to tackle the NP-Hard problem. The unique contribution of this paper is to provide fuzzy processing time in all stages of surgery as well as to introduce a metaheuristic approach from ACO family so as to solve the $f$ SCS problem for the first time. The criterion of the problem was to minimize fuzzy makespan. Our methodology was based on ACO algorithm; we developed a fuzzy two-level AS and introduced a new algorithm, namely $f$ AS. To illustrate our methodology, we provided the data on three case tests; each case comprised three problems with different sizes. The sizes of the problems were changed by increasing duration of surgery, number of surgical cases, and the number of required resources for all stages of surgery. First, we modeled fSCS by using a mathematical programming model and then, compared our approach with this model for problems of small sizes. On the other hand, we constructed fuzzy schedules with FCFS rule, evaluated both methods by AVR, and compared their 
Table 11. Comparison of the performance of the algorithm with real planning in all the real case problems.

\begin{tabular}{cccc}
\hline Problems & Method & \multicolumn{2}{c}{ Objectives } \\
\cline { 3 - 4 } & & Mean fuzzy makespan & ARV (crisp makespan) \\
\hline Instance 1 & $f$ AS & $(406.8,505.4,613.9)$ & 507.875 \\
& Hospital & & 569 \\
Instance 2 & $f$ AS & $(404.2,458.1,503.5)$ & 455.975 \\
& Hospital & & 537 \\
Instance 3 & $f$ AS & $(623.7,708.9,791.5)$ & 708.25 \\
& Hospital & & 714 \\
Instance 4 & $f$ AS & $(603.2,681.3,753.7)$ & 679.875 \\
& Hospital & & 761 \\
Instance 5 & $f$ AS & $(601.9,670.7,744)$ & 671.825 \\
& Hospital & & 823 \\
& & & 531.25 \\
Instance 6 & $f$ AS & $(479,530,586)$ & 666 \\
& Hospital & & 707.9 \\
& & & \\
Instance 7 & $f$ AS & $(639,709.7,773.2)$ & 778 \\
& Hospital & & 469 \\
& & &
\end{tabular}

Table 12. ANOVA results for ARV (crisp makespan).

\begin{tabular}{cccccc}
\hline Source of variation & DF & SS & MS & F & $\boldsymbol{P}$-value \\
\hline Method & 1 & 254303 & 254303 & 18.75 & 0.000 \\
Error & 158 & 2142369 & 13559 & & \\
Total & 159 & 2396672 & & & \\
\hline
\end{tabular}

performances. Moreover, real data were provided from a private hospital in Isfahan to evaluate the proposed algorithm. It was observed that our proposed $f \mathrm{AS}$ algorithm outperformed $f$ FCFS as well as the hospital planning. Here are some directions as opportunities for future research in this area. Emergency cases as patients can be taken into account in the problem and a new ACO algorithm can be constructed in order to solve online surgical case scheduling in real world. On the other hand, building a new fuzzy ACO algorithm for bi-objective surgical case scheduling problem can be a novel path in future research. In this way, our future research goal will be to extend a fuzzy two-level ant system in order to solve fuzzy bi-objective surgical case scheduling problem.

\section{Acknowledgements}

The authors are sincerely grateful to the anonymous referees for their helpful comments and constructive suggestions.

\section{References}

1. Vijayakumar, B., Parikh, P.J., Scott, R., Barnes, A., and Gallimore, J. "A dual binpacking approach to scheduling surgical cases at a publicly-funded hospital", European Journal of Operational Research, 224, pp. 583-591 (2013).

2. Zhao, Z. and Li, X. "Scheduling elective surgeries with sequence-dependent setup times to multiple operating rooms using constraint programming", Operations Research for Health Care, 3(3), pp. 160-167 (2014).

3. Denton, B., Viapiano, J., and Vogl, A. "Optimization of surgery sequencing and scheduling decisions under uncertainty", Health Care Management Science, 10(1), pp. 13-24 (2007).

4. Atighehchian, A. "Stochastic operating room schedul- 
ing", PhD Thesis, Dep. Indsturial Engineering, Tarbiat Modares University, Iran, Tehran (2011).

5. Guerriero, F. and Guido, R. "Operational research in the management of the operating theatre: a survey", Health Care Manag Sci, 14, pp. 89-144 (2011).

6. Zakaria, Y. Abdelrasol, Z.Y., Harraz, N., and Eltawil, A. "A proposed solution framework for the operating room scheduling problems", Proceedings of the World Congress on Engineering and Computer, 2, pp. 11491157 (2013).

7. Samudra, M., Van Riet, C., Demeulemeester, E., Cardoen, B., Vansteenkiste, N., and Rademakers, F.E. "Scheduling operating rooms: achievements, challenges and pitfalls", $J$ Sched, 19(5), pp. 493-525 (2016).

8. Dellaert, N. and Jeunet, J. "A variable neighborhood search algorithm for the surgery tactical planning problem", Computers and Operation Research, 84, pp. 216-225 (2017).

9. Magerlein, J.M. and Martin, J.B. "Surgical demand scheduling: A review", Health Service Research, 13, pp. 418-433 (1978).

10. Fei, H., Meskens, N., and Chu, C. "A planning and scheduling problem for an operating theatre using an open scheduling strategy", Computers \& Industrial Engineering, 58, pp. 221-230 (2010).

11. Adan, I., Bekkers, J., Dellaert, N., Jeunet, J., and Vissers, J. "Improving operational effectiveness of tactical master plans for emergency and elective patients under stochastic demand and capacitated resources", European Journal of Operational Research, 213, pp. 290-308 (2011).

12. Marques, I., Captivo, M.E., and Vaz Pato, M. "Scheduling elective surgeries in a Portuguese hospital using a genetic heuristic", Operations Research for Health Care, 3(2), pp. 59-72 (2014).

13. Marques, I. and Captivo, M.E. "Bicriteria elective surgery scheduling using an evolutionary algorithm", Operations Research for Health Care, 7(24), pp. 14-26 (2015).

14. Aringhieri, R., Landa, P., Soriano, P., Tànfani, E., and Testi, A. "A two level metaheuristic for the operating room scheduling and assignment problem", Computers \& Operations Research, 54, pp. 21-34 (2015).

15. Guido, R. and Conforti, D. "A hybrid genetic approach for solving an integrated multi-objective operating room planning and scheduling problem", Computers and Operation Research, 87, pp. 270-282 (2017).

16. Pham, D.N. and Klinkert, A. "Surgical case scheduling as a generalized job shop scheduling problem", European Journal of Operational Research, 185, pp. 10111025 (2008).
17. Ghazalbash, S., Sepehri, M.M., Shadpour, P., and Atighehchian, A. "Operating room scheduling in teaching hospitals", Advances in Operations Research, 2012 (2012).

18. Davila, M.P., Centeno, G., Fabri, P., Laurentis, K., Reeves, K., and Weng, M. "A methodology for scheduling operating rooms under uncertainty", PhD Thesis, University of South Florida (2013).

19. Meskens, N., Duvivier, D., and Hanset, A. "Multiobjective operating room scheduling considering desiderata of the surgical team", Decision Support Systems, 55, pp. 650-659 (2013).

20. M'Hallah, R. and Al-Roomi, A.H. "The planning and scheduling of operating rooms: A simulation approach", Computers \& Industrial Engineering, 78, pp. $235-248$ (2014).

21. Xiang, W., Yin, J., and Lim, G. "An ant colony optimization approach for solving an operating room surgery scheduling problem", Computers \& Industrial Engineering, 85, pp. 335-345 (2015).

22. Saadouli, H., Jerbi, B., Dammaka, A., Masmoudi, L., and Bouaziz, A. "A stochastic optimization and simulation approach for scheduling operating rooms and recovery beds in an orthopedic surgery department", Computers \& Industrial Engineering, 80, pp. 72-79 (2015).

23. van Essen, J.T., Hurink, J.L., Hartholt, W., and van den Akker, B.J. "Decision support system for the operating room rescheduling problem", Health Care Manag Sci, 15(4), pp. 355-372 (2012).

24. Erdem, E., Qu, X., and Shi, J. "Rescheduling of elective patients upon the arrival of emergency patients", Decision Support Systems, 54, pp. 551-563 (2012).

25. Fügener, A., Hans, E.W., Kolisch, R., Kortbeek, N., and Vanberkel, P.T. "Master surgery scheduling with consideration of multiple downstream Units", European Journal of Operational Research, 239, pp. 227-236 (2014).

26. Cardoen, B., Demeulemeester, E., and Beliën, J. "Operating room planning and scheduling: A literature review", European Journal of Operational Research, 201, pp. 921-932 (2010).

27. Lee, S. and Yih, Y. "Reducing patient-flow delays in surgical suites through determining start-times of surgical cases", European Journal of Operational Research, 238, pp. 620-629 (2014).

28. Marques, I. and Captivo, E.M. "Different stakeholders's perspectives for a surgical case assignment problem: deterministic and robust approaches", European Journal of Operational Research, 261(1) , pp. 260-278 (2017).

29. Van Riet, C. and Demeulemeester, E. "Trade-offs in operating room planning for electives and emergencies: A review", Operations Research for Health Care, 7, pp. 52-69 (2015). 
30. Lamiri, M., Xie, X., Dolgui, A., and Grimaud, F. "A stochastic model for operating room planning with elective and emergency demand for surgery", Eur $J$ Oper Res, 185(3), pp. 1026-1037 (2008).

31. Lamiri, M., Grimaud, F., and Xie, X. "Optimization methods for a stochastic surgery planning problem", Int J Prod Econ, 120(2), pp. 400-410 (2009).

32. Saremi, A., Jula, P., ElMekkawy, T., and Wang, G.G. "Appointment scheduling of outpatient surgical services in a multistage operating room department", Int. J. Production Economics, 141, pp. 646-658 (2013).

33. Mateus, C., Marques, I., and Captivo, M.E. "Local search heuristics for a surgical case assignment problem", Operations Research for Health Care, 17, pp. 71-81 (2018).

34. Dubois, D., Fargier, H., and Fortemps, P. "Fuzzy scheduling: Modelling flexible constraints vs. coping with incomplete knowledge", European Journal of Operational Research, 147, pp. 231-252 (2003).

35. Gonzalez-Rodriguez, I., Vela, C.R., Puente, J., and Hernandez-Arauzo, A. "Improved local search for job shop scheduling with uncertain durations", Proceedings of the Nineteenth International Conference on Automated Planning and Scheduling, pp. 124-131 (2008a).

36. Gonzalez-Rodriguez, I., Puente, J., Vela, C.R., and Varela, R. "Semantics of schedules for the fuzzy jobshop problem", IEEE Transactions on Systems Man and Cybernetics - Part A: Systems and Humans, 38, pp. 655-666 (2008b).

37. Palacios, J.J., Vela, C.R., Puente, J., and GonzalezRodriguez, I. "Hybrid cooperative coevolution for fuzzy flexible job shop scheduling problems", Proceedings of Erofuse, pp. 199-206 (2013).

38. Lei, D.M. "Co-evolutionary genetic algorithm for fuzzy flexible job shop scheduling", Appl. Soft Comput., 12(8), pp. 2237-2245 (2012).

39. Xu, Y., Wang, L., Wang, S., and Liu, M. "An effective teaching-learning-based optimization algorithm for the flexible job-shop scheduling problem with fuzzy processing time", Neurocomputing, 148, pp. 26-35 (2015).

40. Liu, B., Fan, Y., and Liu, Y. "A fast estimation of distribution algorithm for dynamic fuzzy flexible jobshop scheduling problem", Computers \& Industrial Engineering, 87, pp. 193-201 (2015).

41. Lin, J. "A hybrid biogeography-based optimization for the fuzzy flexible job-shop scheduling problem", Knowledge-Based Systems, 78, pp. 59-74 (2015).

42. Guinet, A. and Chaabane, S. "Operating theatre planning", International Journal of Production Economics, 85(1), pp. 69-81 (2003).

43. Augusto, V., Xie, X., and Perdomo, V. "Operating theatre scheduling with patient recovery in both operating rooms and recovery beds", Computers \& Industrial Engineering, 58(2), pp. 231-238 (2010).
44. Pinedo, M., Scheduling: Theory, Algorithms and Systems, Prentice-Hall, Ed., 3th Edn., New Jersey, Englewood Cliffs (2008).

45. Sakawa, M. and Kubota, R. "Fuzzy programming for multiobjective job shop scheduling with fuzzy processing time and fuzzy due date through genetic algorithms", Eur. J. Oper. Res., 120(2), pp. 393-407 (2000).

46. Demir, Y. and Isleyen, S.K. "Evaluation of mathematical models for flexible job-shop scheduling problems", Applied Mathematical Modelling, 37, pp. 977988 (2013).

47. Ozguven, C., Ozbakır, L., and Yavuz, Y. "Mathematical models for job-shop scheduling problems with routing and process plan flexibility", Applied Mathematical Modelling, 34, pp. 1539-1548 (2010).

48. Noori-Darvish, S., Mahdavi, I., and Mahdavi-Amiri, $\mathrm{N}$. "A bi-objective possibilistic programming model for open shop scheduling problems with sequencedependent setup times, fuzzy processing times, and fuzzy due dates", Applied Soft Computing, 12, pp. 1399-1416 (2012).

49. Dorigo, M., Maniezzo, V., and Colorni, A. "Positive feedback as a search strategy", Technical Report 91016, Dip. Elettronica, Politecnico di Milano, Italy (1991).

50. Dorigo, M., Maniesso, V., and Colorni, A. "The ant system: optimization by a colony of cooperating agents", IEEE Trans. Systems Man Cybernet, Part B, 26, pp. 29-42 (1996).

51. Kuo, R.J., Wibowo, B.S., and Zulvia, F.E. "Application of a fuzzy ant colony system to solve the dynamic vehicle routing problem with uncertain service time", Applied Mathematical Modelling, 40(23-24), pp. 999010001 (2016).

\section{Biographies}

Reza Behmanesh is currently a $\mathrm{PhD}$ candidate in Industrial Engineering with specialty in Operation research and System Engineering at Science and Research Branch, Islamic Azad University, Tehran, Iran. He received his BSc degree in Mining Engineering from Isfahan University of Technology, Isfahan, Iran, in 2001 and his MSc degree in Industrial Engineering from Yazd University, Yazd, Iran, in 2010. He has three years of experience in EORC as a planner in maintenance workshop. His current research interests are swarm and evolutionary computations, scheduling, and optimization techniques.

Mostafa Zandieh received his BSc degree in Industrial Engineering from Amirkabir University of Technology, Tehran, Iran, in 1998, and MSc degree in Industrial Engineering from Sharif University of Technology, Tehran, Iran, in 2000. He also obtained his PhD degree 
in Industrial Engineering from Amirkabir University of Technology, Tehran, Iran, in 2006. Currently, he is an Associate Professor in Industrial Management Department at Shahid Beheshti University, Tehran, Iran. His research interests are production planning and scheduling, financial engineering, quality engineering, applied operations research, simulation, and artificial intelligence techniques in the areas of manufacturing systems design.

Seyed Mohammad Hadji Molana was graduated with BSc from Sharif University of Technology, and MSc and PhD from Amirkabir University of Technology in Iran. His research interests are in supply chain management, advanced inventory control, and production planning. 